\title{
An Experiment to Prove the Effect of Low-Level Magnetic Fields Resulting from Ionospheric Changes on Humans
}

\author{
M. Hanzelka ${ }^{1}$, J. Dan ${ }^{2}$, M. Šlepecky ${ }^{4}$, V. Holcner ${ }^{3}$, P. Dohnal ${ }^{1}$, R. Kadlec ${ }^{1}$ \\ ${ }^{1}$ Department of Theoretical and Experimental Electrical Engineering, Brno University of Technology, Technická 3082/12, \\ 61600 Brno, Czech Republic \\ xhanze09@feec.vutbr.cz,tel.:+420541146280,fax: +420541146276 \\ ${ }^{2}$ Rector's Office, Personnel Management Office, Masaryk University, Žerotínovonám. 9, 60177 Brno, Czech Republic \\ dan@rect.muni.cz, tel.: +420549493455 \\ ${ }^{3}$ Faculty of Economics and Management, University of Defence, Kounicova 65, 66210 Brno, Czech Republic \\ vladan.holcner@unob.cz, tel.+420973444956 \\ ${ }^{4}$ Constantine the Philosopher University in Nitra, Trieda A. Hlinku 194974 Nitra, Slovak Republic \\ milos.slepecky@gmail.com,tel.+421903500887
}

\begin{abstract}
The investigation presented in the paper was performed in the laboratories of the Department of Theoretical and Experimental Electrical Engineering, Faculty of Electrical Engineering and Communication, Brno University of Technology, between April22 and June $26,2014$. We examined a homogeneous sample of male and female participants comprising a total of 49 persons aged 19 to 26 . The time required for the measurement of psychophysiological parameters corresponded to 19 minutes, encompassing five stages: Basic (5 mins.), Color (2 mins.), Rest (5 mins.), Math (2 mins.), and Rest (5 mins.). All the measuring cycles were carried out using a BioGraph Infiniti device (Thought Technology, Ltd.). Generally, the impact of the environment upon living organisms constitutes a crucial problem examined by today's science. In this context, the present article describes the results of an investigation focused on ionosphere parameter variation and its role in the basic function of the nervous system. The discussed research concentrates on the measurement and detection of changes in the region of very low electromagnetic field frequencies; the authors introduce and verify related theoretical and experimental procedures to define the effects that influence brain activity and the cardiovascular system.
\end{abstract}

Keywords: Ultra low Frequency, Super Low Frequency, Extremely Low frequency, BioGraph Infiniti, Schumann resonances, heart coherence.

\section{INTRODUCTION}

The low-level measurement of low frequencies $(0.01$ $10 \mathrm{~Hz}$ ) performed to evaluate the effect of magnetic fields on the human organism can be regarded as an interdisciplinary branch of science that embraces different types of research. In this context, it is important to consider applied research disciplines, such as the measurement and radar technology in the following ranges: the ULF (Ultra Low Frequency Band: $300 \mathrm{~Hz}-3 \mathrm{KHz}$ ), SLF (Super Low Frequency Band: $30 \mathrm{~Hz}-300 \mathrm{~Hz}$ ), and ELF (Extremely Low Frequency Band: $0.1 \mathrm{~Hz}-30 \mathrm{~Hz}$ ) [3]. The current status of knowledge in the given field is relatively unsatisfactory, and certain hasty conclusions have been made and subsequently applied even in hygienic standards. An example of such standards is the guideline issued by the Council of Europe and implemented by ICNIRP [4] in 1999 to establish the boundary values of magnetic flux in relation to the speed of magnetic field variations for very slowly changing currents. More concretely, this guideline introduces the value of $50 \mathrm{mT} / \mathrm{s}$ as the maximum magnetic flux change acceptable in an environment having a variable magnetic field at the frequency of $1 \mathrm{~Hz}$ and characterized by permanent presence of humans. This value is many million times higher than the largest changes hitherto measured during processes referred to as magnetic storms, in which the Earth was exposed to charged particles from the Sun.

The paper proposes an experiment involving comprehensive coverage of measurable parameters of the human body in a homogeneous sample of participants; the participants are monitored repeatedly, and the relevant parameters are evaluated with respect to external magnetic field changes. The generation and effects on the human organism of low-level magnetic fields induced by solar activity were described previously, [3] and [4].

According to the conclusions of the secondary research, there is mutual interaction between low-level electric or 
magnetic fields irradiated by both humans and the geomagnetic system. We present a portion of the research conducted in this province at the Department of Theoretical and Experimental Electrical Engineering (DTEEE), Faculty of Electrical Engineering and Communication (FEEC), Brno University of Techology (BUT); the investigation exploits the current knowledge of low-level magnetic fields generated by the geomagnetic mechanism and the solar system. The attention is centered on examining the effect of solar activity-induced changes in Earth's magnetic system. In this context, the research also focuses on proving the existence of that effect as a result of geomagnetic storms, which substantially influence low-level magnetic fields affecting the human organism, including its behavioral patterns and decision-making [5]. The detection of changes and disturbances in the geomagnetic field could be performed by means of the Schumann resonances. Until recently, the relevant oscillation was at the average yearly frequency of fsch $=7.83 \mathrm{~Hz}$; this frequency changes in consequence of the impact exerted by phenomena such as the solar wind or greenhouse gases. In 1953, Professor W.O.Schumann of the Technical University of Munich, Germany [6] found out that the cavity between the ionosphere and Earth's surface could be interpreted as a spherical resonator.

\section{SUBJECT \& METHODS}

The laboratory-based research comprising a homogeneous sample of 49 subjects (men and women aged 19 to 25) began on April 22, 2014 and lasted until June 26, 2014 [7]. The young age and homogeneity characteristic of the group of participants led to stable cognitive competencies such as working memory, selective attention, multitasking, task switching, response monitoring, and error detection. Nearly all of these functions show an age-related decline [8]. The total time required for the examination of the psychophysiological parameters of a subject corresponded to 19 minutes. We used a Nickelodeons Infiniti [9] (Thought Technology, Ltd.) unit to perform the entire task, and the measurement proper involved five phases: Rest; Color; Rest; Math; and Rest. At the Color stage, a special (Stroop) test was utilized to acquire the psychophysiological responses of each subject to a load on their organism. Generally, this tool demonstrates that a person performing the given task can be easily distracted due to their automatic reactions and habits; the procedure is named after John Ridley Stroop (1897 1973), the American psychologist who first described the phenomenon in 1929 [10]. In the Math phase, then, the participants were asked to progressively subtract the number 7 from the initial value of 1081 , and we examined the psychophysiological stress generated during such quiet countdown. The total number of measurements was 210 , with the average of 4.29 per participant. The relationship between the above total count and the number of subjects who completed the task is shown in Table 1. The laboratory was configured to facilitate the measurement of comparable parameter values, namely constant temperature, noise, humidity, lighting, concentration of positive and negative ions, and homogenized geomagnetic field component (Fig.3.c)).

\section{RESULtS}

A major part of the laboratory research consisted of largescale collection of psychological data from all participants (respondents) via several psychological tests, including an ASS-SYM test; this tool measures a respondent's sensitivity to changes in the transition from load to relax. All the results were then correlated with the psychophysiological measurements.

Table 1. The relationship between the number of completed measurements and the number of respondents.

\begin{tabular}{|c|c|}
\hline $\begin{array}{c}\text { Number of } \\
\text { respondents }\end{array}$ & $\begin{array}{c}\text { Number of } \\
\text { measurements }\end{array}$ \\
\hline 4 & 1 \\
\hline 1 & 2 \\
\hline 4 & 3 \\
\hline 12 & 4 \\
\hline 24 & 5 \\
\hline 4 & 6 \\
\hline 49 & SUM \\
\hline
\end{tabular}

The measurement comprised three relaxation and two stress phases, with an emphasis on intensive psychological stress in the participants. The stress stages were induced using a low-level electromagnetic field generator, an amplifier, and Helmholtz coils to interact with the EEG brain waves of the participant involved. The resulting lowlevel field enabled us to simulate the effect of solar activity changes as an additional offset to the related real intensity indicators prepared for each day by NASA. The field strength in the Helmholtz coil was set to the level of the terrestrial magnetic field, and the major parameters were as follows: pulse $\mathrm{f}=1 \mathrm{kHz}$; start $=100 \mathrm{~ns}$; wavelength $\lambda=$ $50.4 \mathrm{~km}$ and $\lambda / 2=25.2 \mathrm{~km} ; \mathrm{T}=168 \mu \mathrm{s}$; amplitude $1 \mathrm{~V}$ on the generator- amplified, out of coil; $B m a x=60 \mu \mathrm{T}$, according to Fig.1. The indicated spectrum is a swept one, between $0.01 \mathrm{~Hz}$ and $30 \mathrm{~Hz}$.

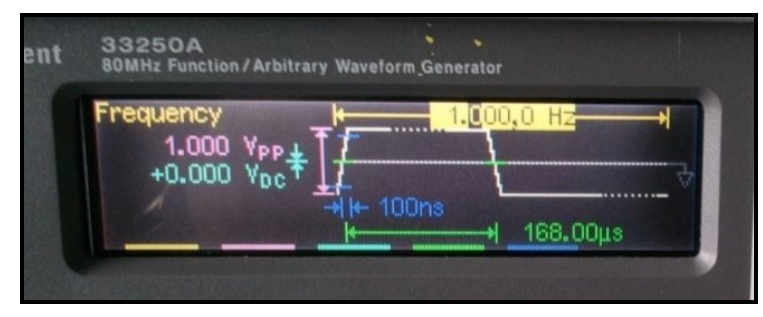

Fig.1. The waveform to offset field strength in the Helmholtz coil.

Fig.1. and Fig.2. illustrate the stimulation and measurement apparatus, and Fig.3. presents shots of the BioGraph Infiniti measurements.

In the course of the experimental research, a large volume of psychophysiological data were measured with the BioGraph Infiniti software; the actual measurement fundamentally exploited the states of relaxation and mental load placed upon each participant, whose mental condition was subsequently evaluated by means of psychological tests. 


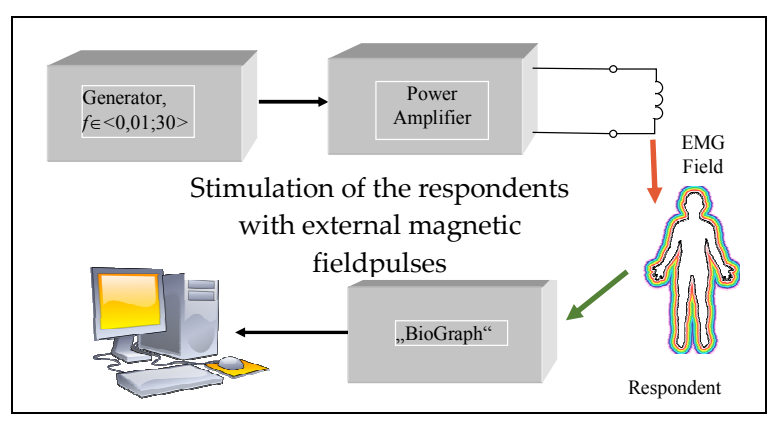

Fig.2. A scheme of the measurement and simulation apparatus.

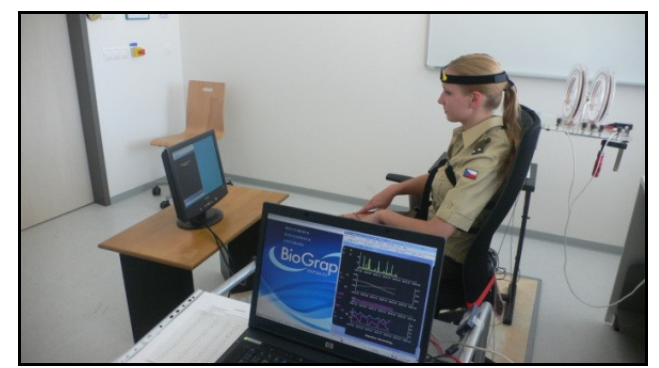

a)

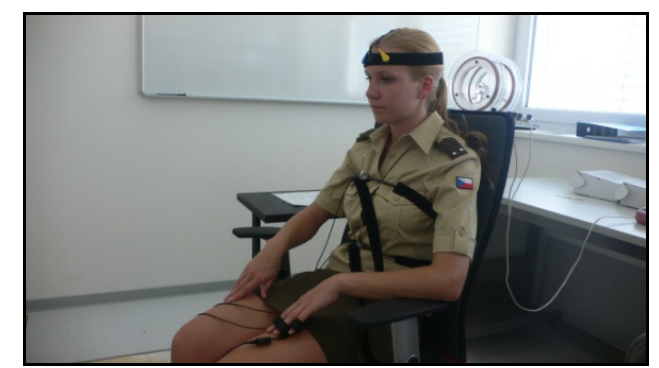

b)

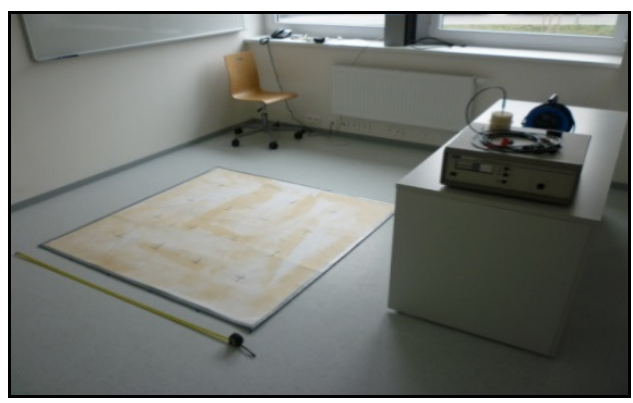

c)

Fig.3. The documentation shots from the laboratory measurements, a), b); the room with a geomagnetically stable magnetic field component, c).

The determined states can be expressed qualitatively. Based on the obtained information, we created a large, unique set of correlational dependencies existing between psychophysiological parameters of the human organism (or the related qualitative psychological measurement) and the intensity of solar activity.

Solar wind particles captured by Earth's magnetic field travel, especially in polar areas, along magnetic lines up to the upper layers of the atmosphere, where they - together with ultraviolet radiation from the Sun - excite and ionize neutral atoms. The excited atoms then emit a typical glow to form the well-known aurorae, and these ionized atoms are seized by the magnetic lines and move freely along them. Through this description, we have merely summarized general facts commonly exploited and referred to by various researchers. Methods and procedures for the measurement of ionosphere changes are currently available [11], Fig.3. In this context, secondary investigation of earlier observations may enable us to point out the connection between magnetic field changes and the social behavior of a group of humans. The corpus of specialized research on the problem comprises a large number of fundamental papers and monographs; interestingly, the achievements of the author of [12], Rollin McCraty, could also be of importance in many respects. McCraty gradually analyzed the impact of the environment on the physical, mental, emotional, and spiritual coherence of an individual, and he also outlined the relationship between these aspects and the cardiovascular system with its resonant frequency of $0.1 \mathrm{~Hz}$ (the ELF band of between 0.04 and $0.26 \mathrm{~Hz}$, a precondition of cardiac coherence). Tchijevsky, then, found out that $80 \%$ of the most significant events in human history occurred within an approximately five-year segment of solar activity, as illustrated in Fig.4., [12].

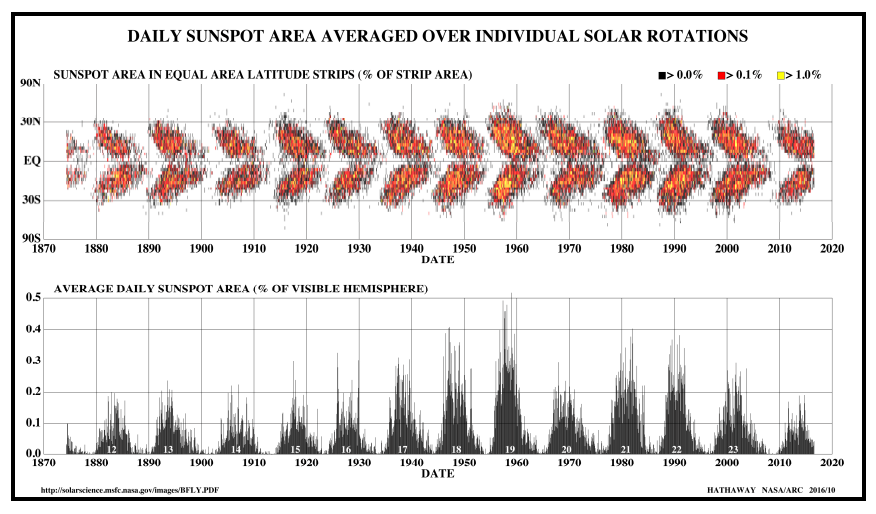

Fig.4. The values of solar activity since 1874, [12].

With respect to these aspects, however, no investigation has been performed thus far with a homogeneous group of selected human subjects to prove the long-term impact of changes in Earth's magnetic field and to eliminate any possible major influence of other factors.

During our experimental research, solar activity was monitored via NASA-indicated data: values acquired from daily solar activity measurements are collected by the agency for scientific purposes. Relevant graphs within this analysis present the solar activity record characterizing the activity period observed at the DTEEE, FEEC, Brno University of Technology.

The parameter of the Sun reflects the actual influence of solar wind particles, which - according to the velocity of the wind - bombard Earth's atmosphere, with a delay of between 2 and 8 days. A related study proposes that solar winds could be interpreted as collision less plasma moving 
radially from the Sun at speeds oscillating between 300 and $800 \mathrm{~km} / \mathrm{s}$. The concentration of the wind varies from 1 to $10 \mathrm{~cm}-3$, and its temperature ranges between $1-30 \mathrm{eV}$. The mass spectrum comprises mainly protons ( $96 \%)$ and helium nuclei ( $\sim \%$ ); the proportion of heavier elements is, for our purposes, negligible. In order to facilitate the measurement of solar wind parameters (or, more concretely, the proton component), a suitable detector was designed to operate from the orbit.

\section{Hypotheses:}

H0. The low-level magnetic fields generated by solar activity exert a negative impact on the human organism and influence human behavior and decision-making.

H1. The mutual interaction between the low-level magnetic and electromagnetic fields of a human subject and the lowlevel magnetic fields induced by geomagnetic changes constitutes a significant phenomenon.

H2. Solar activity, the resulting manifestations of geomagnetic storms, and their comprehensive effect on the human organism as regards economic behavior and decision-making are directly interrelated factors.

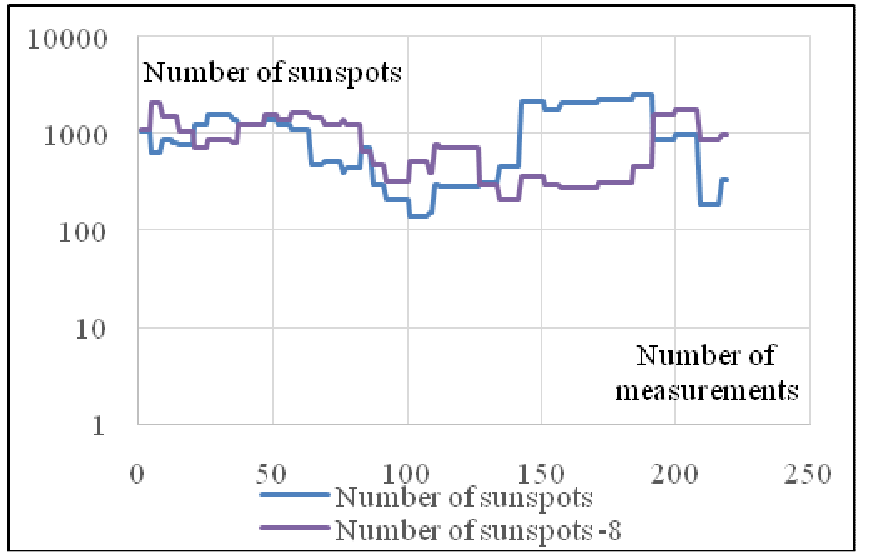

Fig.5. The progression of solar activity during the experimental research specified in chapter 3 (investigation performed between April 22 and June 26, 2014). Source: [13].

\section{Data Analysis:}

\section{Static input psychological prerequisites}

Before the actual commencement of the research, the 49 participants were assessed with the ASS-SYM and MBTI psychological testing methods. At the initial stage, before analyzing the methodology, we consider it important to introduce a brief definition of health as an underlying concept within our investigation. According to World Health Organization, health is "a state of complete physical, mental and social well-being and not merely the absence of disease or infirmity" [14]. This definition then leads us to a rather narrow domain of psychological methodology, namely self-assessment scales. With respect to these instruments, it is possible to accentuate the ASS-SYM, a representative method designed by the German psychologist Gunther Krampen [15]. The ASS-SYM, or Änderungssensitive Symptom Liste, constitutes an index of change-sensitive symptoms related to relaxed perception and experiencing, life satisfaction, mental load, problems, and other aspects outlined in [15]. The list comprises 48 items, within which each participant expresses themselves in four degrees. As proposed, the symptoms are sensitive to changes during autogenic training and progressive relaxation. The ASS-SYM has been verified as a part of the overall evaluation of the quality and effect of various therapeutic procedures; its advantage consists in that, unlike some other techniques, the discussed instrument does not cause excessive stress to the examined subject or bring any additional difficulties. The subscales relate to 6 areas (each comprising 8 items), and these domains involve several specific activities or elements: the examination and influencing of "intrinsic" mental processes such as the interpretation and assessment of the self and the environment; the core beliefs and assumptions of who I am and what the world around me is like; and determining the interrelationships or mutual impact between emotional experiences and cognitive mechanisms (or, in plain words, finding out how the brain and the heart influence each other). By definition, the areas concerned are presented as follows:

- $\quad$ Physical and mental exhaustion (such as "sleep disorder and falling asleep difficulty“");

- Nervousness and mental tension ("internal stress, nervousness"');

- Psychophysiological dysregulation ("inappetence“);

- Behavioral and performance-related disorder ("performance anxiety prior to tests, exams, and similar situations ");

- $\quad$ Self-control difficulties ("head aches or pressures");

- General symptoms and problems ("indecisiveness, decision-making difficulty").

The initial psychological examination as a component of the wider test of the impact of geomagnetic field changes on a human being was attended by 38 from the total of 49 participants, and the obtained results are indicated in Fig.6. below.

In the course of the measurement, the majority of participants (mostly of military background) exhibited physical and mental exhaustion, behavioral and performance-related disorder, and burden of pain. Nine participants were affected by simultaneous action of all the three effects; interestingly, in six participants out of these nine we detected concurrent affection by 5 from the aboveoutlined 6 effects. This set of participants will be hereafter referred to as emotionally labile, EL. Even though a majority of the symptoms clearly manifested themselves at this stage, none of the subjects exhibited psychophysiological dysregulation.

The follow-up phase of the research consisted of MBTIbased personality testing. In spite of substantial character differences, the participants can be classified into 16 basic categories, each comprising a set of subjects with certain identical traits; thus, we respect the system of personality types founded by Carl Jung, further developed by American researchers Katharine Cook Briggs and Isabel Briggs Myers, and refined to its current forms (as regards, for example, the nomenclature) by later 20th century scholars [17]. 
The relevant classes are determined via the Myers-Briggs Type Indicator (MBTI); when using this instrument, we adopted - in accordance with the above references personality type denomination variants that best suited our purposes, and the applied typology then was as follows:

The type indicator (MBTI) was applied in only 44 out of the total of 49 respondents; a portion of these subjects did not participate in the initial psychological testing and attended merely the laboratory measurements. The MBTI test was performed prior to the measurement cycle via psychological testing of each participant.

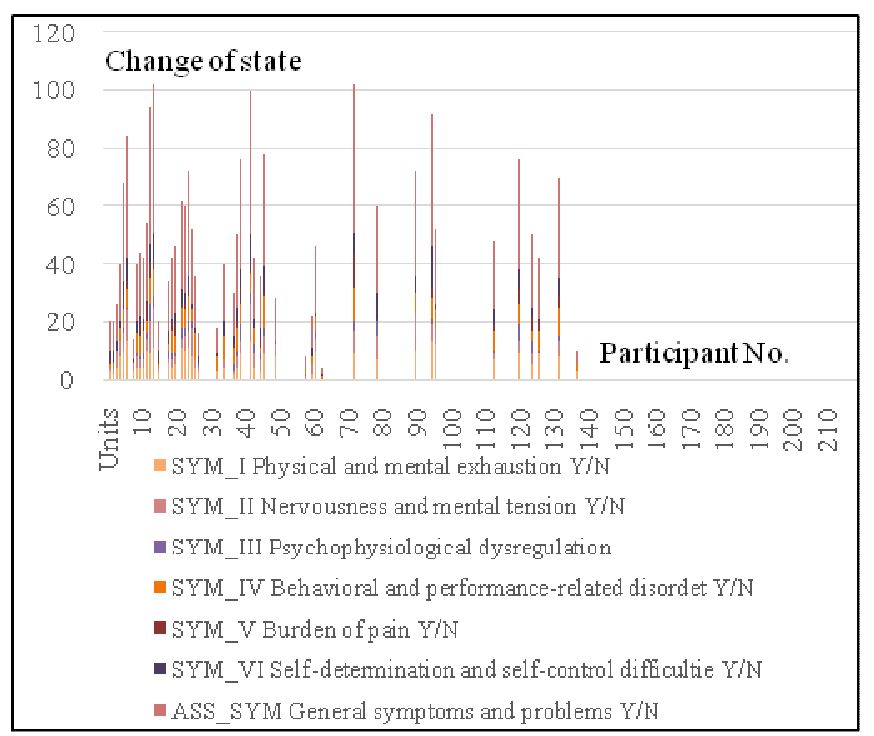

Fig.6. The assessment performed via the ASS-SYM instrument. Source: [16].

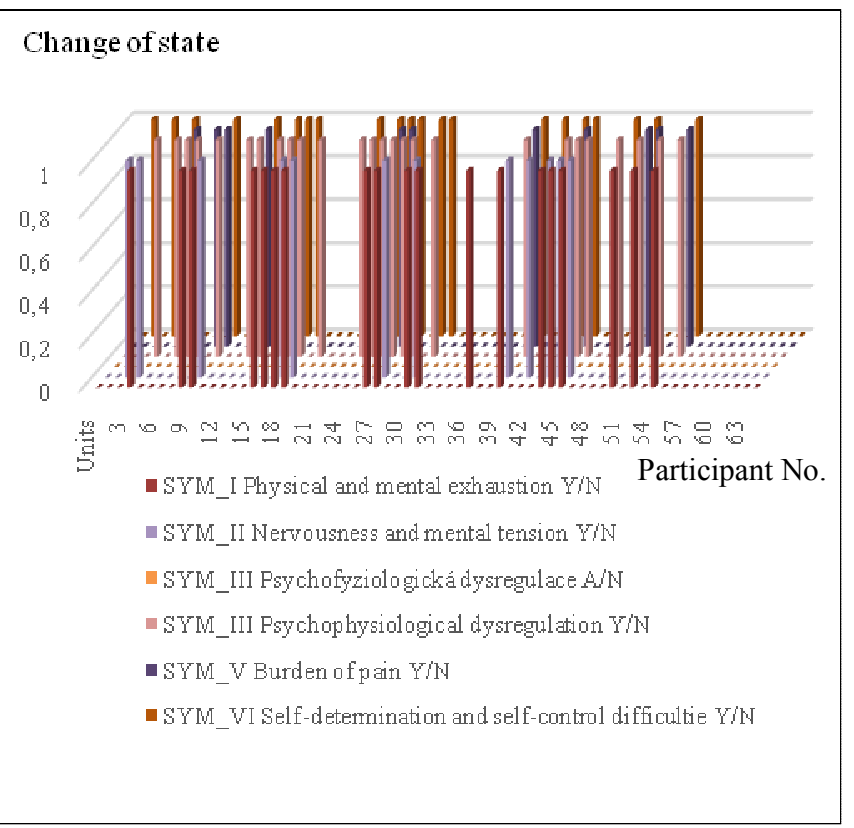

Fig.7. The cyclic change of the mental state in the three respondents specially observed during the experimental research. [16].
In the given context and as already outlined, from the general and theoretical perspectives, we investigate the influence of geomagnetic fields in relation to "intrinsic" human mental processes, such as the interpretation and evaluation of the self and the environment, core beliefs and assumptions of who I am and what the world around me is like, and we also focus on defining the mutual interaction between emotional experiences and cognitive processes, between the brain and the heart [18].

\section{Dynamic gradual psychological prerequisites}

Each psychophysiological measurement cycle was preceded by a Lüscher color test session. The Lüscher test concept comprises 8 color cards (blue, green, red, yellow, violet, brown, grey, and black) combinable into 40,320 possible answers. The main advantage of the instrument consists in its hidden validity: as the participant does not know the purpose of this particular test and cannot estimate what is being defined by it, they are not able to suitably modify their answers in advance to fit the presumed or desired result. Applicable criticism targeting the alleged insufficient reliability of the method is analyzed by, for example, the main author of papers [19], [20], and [21], who proposes that the test captures the dynamics of mental states and that "the determination of reliability in the sense of consistency is not feasible" owing to the structure of the instrument. The repeated use of the Lüscher color test enabled us to reveal progressively the actual mental state of the respondents prior to the main psychophysiological measurement, especially as regards the following parameters:

$\mathrm{S}$ - deteriorated mental condition; A - anxiety; B emotional discomfort; A, B - mental imbalance.

The pattern in Fig.7. shows deteriorated mental condition in three subjects of the EL group; this decrease occurred cyclically during the entire experimental research and in all the monitored variables.

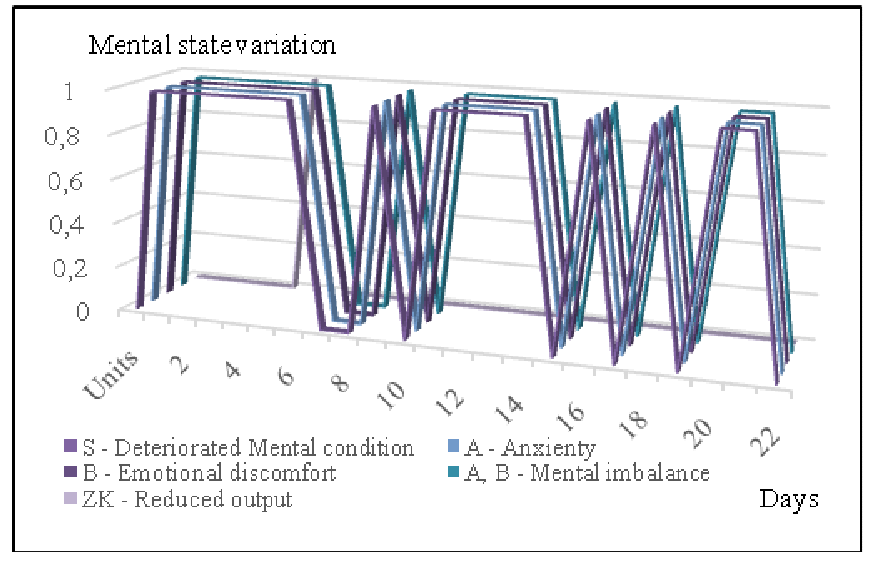

Fig.8. The sample of participants exhibiting reduced performance. Source: [16]. 
Another quantity monitored within the dynamic psychological data was mirror drawing, intended to define the instantaneous performance rate of the subjects. This activity was used to conclude each physiological measurement session. Fig.8. then represents the set of participants who exhibited signs of reduced performance; this group comprises32 out of the 49 respondents participating in the psychophysiological measurement.

Relationship between individual changes in the intensity of solar processes, and the effect of such changes on the skin conductance of examined participants

According to a major reference [22], skin conductance changes are caused by activation of the autonomic nervous system rather than by emotions. Based on the actual needs, however, strong emotions prepare the human organism for an intensive activity; variation in emotions is then accompanied by accelerated breathing, cardiac function, and vascular circulation. This process results in altered skin conductance, from the lowest level in sleep to the highest one attained under conditions such as emotional stress. The present study follows from the assumption that emotional lability in responsive individuals is directly related to and incited by changes of solar activity; conversely, in the same context, participants classified within the preliminary testing cycle as emotionally stable will be generally less sensitive to the discussed variations. The performed measurements propose that, in emotionally responsive individuals, skin conductance depends on changes in the intensity of solar activity (namely, the increase or decrease of such activity) rather than on its amplitude. As illustrated in Fig.9., the variation of solar activity then constitutes an element abetting emotional lability in humans, and this condition manifests itself in the growth of skin conductance. During the laboratory-based measurement of the psychophysiological parameters of the human organism in relation to the intensity of solar activity, we simulated the effects of such activity via using a pulse magnetic field (as already mentioned above) that exhibited the magnetic flux density of $B_{\max }=60 \mu \mathrm{T}$ in the region outside the Helmholtz coil; this field spread out towards the head of a participant, into a homogenized environment (this environment was completed with a special shielding bolster located in the vicinity of a participant, embodying the modification of Earth's inhomogeneity - namely, a quasi-stationary field and the influence of natural radioactivity). At the head of a participant, which was positioned outside the absolutely homogeneous field, we set/measured $B_{\max }=5.0 \mu \mathrm{T}$. The exposure to the given field was invariably triggered in the Color and Math load phases, in two-minute intervals. For the purposes of pulse measurement, identical repeatable conditions were set for all participants. The exposure was eliminated from the Rest stages. The total experiment time allocated to each respondent, including the preparation and connection to the measuring apparatus, equaled 40 minutes; within this period, a participant was exposed to the applied pulse magnetic field for four minutes.
Within the research, we also considered the actual effect of solar activity, assuming the delay of -4 to -8 days according to the relevant solar wind intensity (Fig.5.). The laboratorybased simulation of the conditions enabling the effect of solar activity brought into the measurement an additional gradient of low-level magnetic field changes in different phases of the psychophysiological measurements. Such changes are exemplified, with respect to skin conductance, in Fig.9., which also indicates the action of an offset pulse (twice every two minutes) during the entire forty-minute measurement. To amplify the effect of an external magnetic field resulting from solar activity, we performed the measurement in the lowest lying laboratory of the DTEEE.

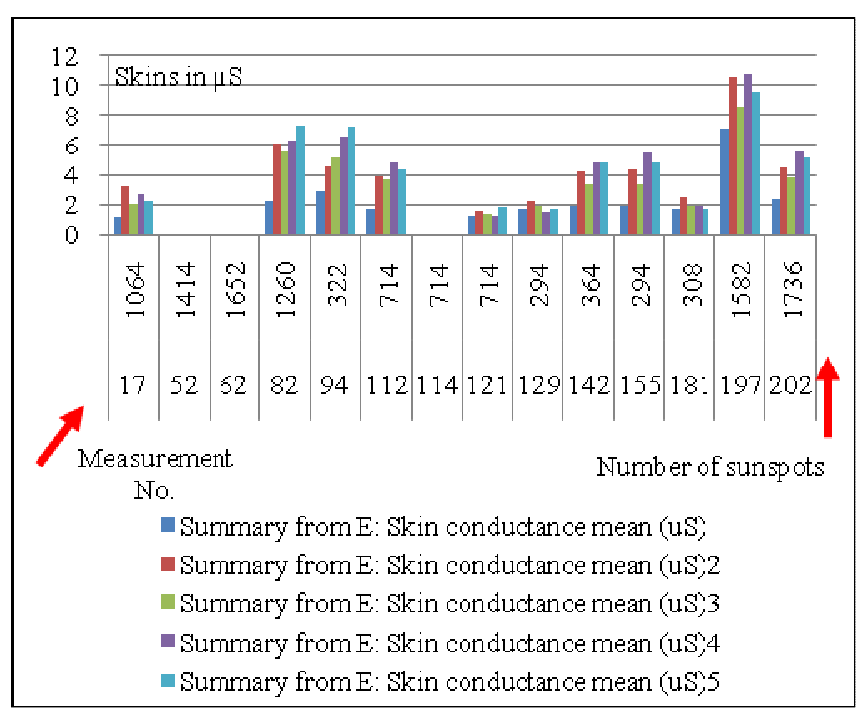

Fig.9. The variation of solar activity constitutes an element abetting emotional lability in humans; this condition manifests itself in the growth of skin conductance [16].

\section{Changes in the intensity of solar activity and their effect on cardiac variability in the sample of participants}

The theoretical basis for the domain of cardiac variability exploits the frequency analysis specified in Table 2.

A relevant reference [23] proposes that heart rate variability (HRV) is considered the neurocardiac function factor which reflects the interaction between the heart, brain, and autonomic nervous system (ANS) dynamics. All HRV parameters are derived from the evaluation of natural heartbeat changes, and HRV as such is substantially more than a mere indicator for cardiac frequency assessment: it reflects the complex interactions between the heart and a high number of bodily systems [24]. The optimum level of variability in key regulation systems of an organism has fundamental meaning for its internal flexibility and adaptability or, by extension, the resistivity and immunity, which embody a healthy coherent function and overall wellbeing. While an excessive amount of instabilities exerts a negative impact on effective physiological functioning and energy usage, a variation too low denotes an energy decrement or cardiac pathologies [25]. 
Table 2. The description and definition of the calculated parameters of the spectral analysis of cardiac frequency variability [25].

\begin{tabular}{|c|c|c|c|c|}
\hline \multicolumn{5}{|c|}{ Frequency band } \\
\hline Description & VLF $[0,02-0,05] \mathrm{Hz}$ & LF $[0,05-0,15] \mathrm{Hz}$ & $\mathrm{HF}[0,15-\mathrm{F}] \mathrm{Hz}$ & Physical unit \\
\hline Spectral power & Power VLF $=\int_{0,02}^{0,05} P S D d f$ & Power $L F=\int_{0.05}^{0,15} P S D d f$ & Power $H F=\int_{0,15}^{F} P S D d f$ & $\mathrm{~ms}^{2}$ \\
\hline $\begin{array}{l}\text { Relative spectral } \\
\text { power }\end{array}$ & Rel. VLF $=\frac{\text { Power VLF }}{\text { Total Power }}$ & Rel. $L F=\frac{\text { Power } L F}{\text { Total Power }}$ & Rel. $\mathrm{HF}=\frac{\text { Power } \mathrm{HF}}{\text { Total Power }}$ & $\%$ \\
\hline $\begin{array}{l}\text { Individual spectral } \\
\text { power ratios }\end{array}$ & VLF/HF $=\frac{\text { Power VLF }}{\text { Power HF }}$ & $L F / H F=\frac{\text { Power } L F}{\text { Power } H F}$ & $V L F / L F=\frac{\text { Power VLF }}{\text { Power LF }}$ & - \\
\hline $\begin{array}{l}\text { Compensation coeffici- } \\
\text { ent for the effect of RR } \\
\text { interval magnitudes }\end{array}$ & $\begin{array}{c}C C V V L F= \\
=\frac{\sqrt{\text { Power VLF }}}{R R} * 100\end{array}$ & $=\frac{\frac{\text { CCVLF }}{\sqrt{\text { Power } L F}}}{R R} * 100$ & $\begin{array}{c}\text { CCVHF }= \\
=\frac{\sqrt{\text { Power HF }}}{R R} * 100\end{array}$ & $\%$ \\
\hline $\begin{array}{l}\text { Total spectral } \\
\text { power }\end{array}$ & Total Power $=\int_{0,02}^{F} P S D d f$ & bo (Power VLF + Power & + Power HF) & $\mathrm{ms}^{2}$ \\
\hline $\begin{array}{l}\text { Mean value of RR } \\
\text { intervals }\end{array}$ & & $R R=\frac{1}{n} \sum_{1}^{\mathrm{n}} R R_{i}$ & & $\mathrm{~ms}$ \\
\hline $\begin{array}{l}\text { Mean value of the power } \\
\text { of sequential differences } \\
\text { of the RR intervals }\end{array}$ & & $D=\frac{1}{n-1} \sum_{1}^{n}\left(R R_{i}-R R_{i-1}\right.$ & & $\mathrm{ms}^{2}$ \\
\hline
\end{tabular}

The quantity or extent of total HRV relate to the presentday lifestyles and are higher in younger people than in older ones [26]. A low HRV constitutes a strong and independent predictor of future health problems, including all mortality causes [27], and it is connected with a number of health conditions [28], [29]. Generally, HRV is also a significant indicator of not only psychological and behavioral resilience but also the ability to adapt oneself effectively to varying social or environmental conditions [30], [31], and HRV quiescent levels are associated with individual cognitive performance differences in tasks that require the use of physical performance functions [31]. Heart rate variability is reflected in the total cardiac power spectrum as a large output increase within low frequency (LF) bands (typically around $0.1 \mathrm{~Hz}$ ) and a decrease at very low frequencies (VLF) or in high frequency (HF) bands [32], [33]. Thus, heart rate variability can be defined as relatively harmonic (sine-wave-like) signals with very narrow and high peak amplitude in the LF region of the HRV power spectrum but without main peaks in the VLF or HF regions. This holds true for the respiratory frequency of about 6 breaths per minute, where the complex ICA is reduced, and the characteristic introduced in the above definition is regarded as the load for the organism and possible training. Such variability then approximately consists in the amplitude ratio LF / (VLF + HF); more specifically, for the above-described problem, variability is observed between 0.04 and $0.26 \mathrm{~Hz}$ of the total power spectrum range. In general terms, the discussed phenomenon constitutes a highly effective functional mode that is closely connected with the expedient use of energy sources and with many health-related advantages. These include six central aspects, namely

1) resetting the sensitivity of the baroreceptors that relate to short-term blood pressure control and increased respiratory efficiency [34];

2) increased vagus nerve afferent stimulation, which participates in the inhibition of signals in sympathetic nerves [34];
3) improved cardiac output in conjunction with enhanced efficiency in fluid exchange, filtering, and absorption between the capillaries and tissue [34];

4) strengthened ability of the cardiovascular system to adapt to circulation requirements;

5 ) increased time synchronization of cells in the whole body (Langhorst et al. 1984); and

6) improved synchronization of the mutual activity of ANS branches [24].

The above elements all result from the overall coherence to energetically enhance the entire system [34]. Another facet of the variability mode is the resonance effect. In this context, applicable mathematical models can be used to demonstrate that the resonant frequency of the human cardiovascular system is determined according to the feedback between the heart and the brain [34]. In humans and a large variety of animals, the resonant frequency of the system corresponds to approximately $0.1 \mathrm{~Hz}$. Based on the proposed details, we can conclude that variability and resonance are typical of the natural physiological effects associated with the heart; these effects constitute and induce positive emotions. The graphs below show a continuous, solar activity-related BVL HF and BLV LF heart variability record of the selected sample of emotionally labile respondents. According to the graphically presented data, a higher emotional load was accompanied by a domination of BVP LF. However, it is not completely clear whether the cause of the higher BVP LF consisted in a changed intensity of solar activity or in the actual emotional character of each respondent within the EL group.

Functions of the ANS are also markedly influenced by respiratory sinus arrhythmia, which is, from the frequency perspective, found in the predominantly weighted spectrum (HF) situated between $0.15-0.4 \mathrm{~Hz}$; the respiratory frequency then correspondingly ranges between 9-24 breaths/min. In the described experimental research, we observed negative breathing, where thoracic respiration dominates. By correlating such negative breathing with solar activity changes, we reach the conclusion that, in the group of emotionally labile respondents, negative breathing manifested itself mainly as a result of a change of the intensity of solar activity without any major influence of the amplitude of such activity.

\section{DISCUSSION / CONCLUSIONS}

During the experimental research, large amounts of quantifiable psychophysiological data based on the states of relaxation and mental load were obtained using the BioGraph Infiniti software. In this context, we also employed psychological tests to determine the mental state of the respondents, and the results of this phase can be expressed qualitatively. These procedures enabled us to form a unique, extensive group of correlational dependencies existing between psychophysiological parameters of the human organism and the intensity of solar activity with corresponding changes of the external electromagnetic field. 
Impact of changes in the intensity of solar activity on skin conductance (skins) in the examined respondents

According to the research presented in [26], skin conductance changes are induced by the activation of the autonomic nervous system rather than by emotions. Strong emotions, however, prepare the human organism for a major activity, depending on the actual need; in the course of an emotional change, the breathing, cardiac activity, and vascular circulation all accelerate. This process then results in changed skin conductance, from the lowest level during sleep to the highest values typical of situations such as emotional stress.

Significantly, this paper as a whole is based on the presumption that the lability of individuals more prone to being influenced by emotional stimuli will be abetted by variation in solar activity; conversely, in this context, those participants who exhibited relatively consistent emotional stability during the pre-research testing will show less dependence on the discussed changes. The measurement results propose that skin conductance in emotionally sensitive individuals depends on variation in solar activity (growth or drop) rather than on its amplitude. According to Fig.10,, a solar intensity change increases the rate of emotional lability in the human organism, thus also causing the relevant skin conductance to grow.

\section{Impact of changes in the intensity of solar activity upon heart variability in the respondents}

The research showed that such correlational dependence manifested itself markedly in skin conductance (or its growth) and heart variability via changes in the $\mathrm{LF} / \mathrm{HF}$ frequency ratio, shown in Fig.11.; domination of the parasympaticus was observed, and thoracic respiration, i.e., negative breathing, prevailed over abdominal respiration.

The evaluation presented herein relates to participants whose psychological test results (those obtained from the ASS-SYM, MBTI, Lüscher-Color-Diagnostik, and mirror drawing) exhibited increased mental lability.

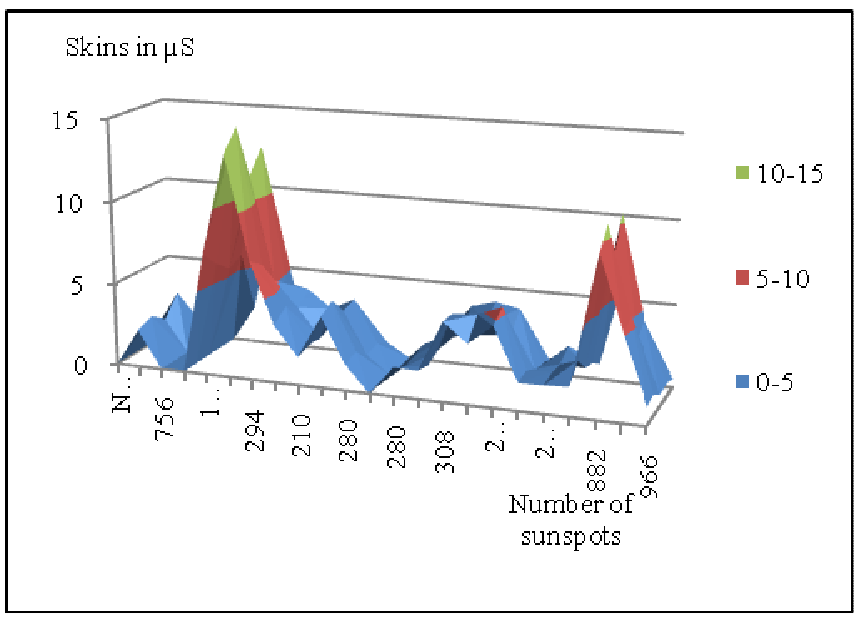

Fig.10. The relationship between skin resistance and solar activity changes in labile individuals. Source: [16].
The follow-up research will continue to evaluate the above-described dependencies in all participants, thus including also those who showed stable or variable mental condition, and the individual procedures will enable us to establish a benchmark for the psychophysiological parameters of healthy, mentally balanced subjects. Further, this benchmark is expected to facilitate mutual comparison between the groups of participants involved in the research.

The investigation of the intensity of solar activity, a phenomenon contingent on changes of solar wind intensity, was performed in the course of using data acquired and classified by NASA.

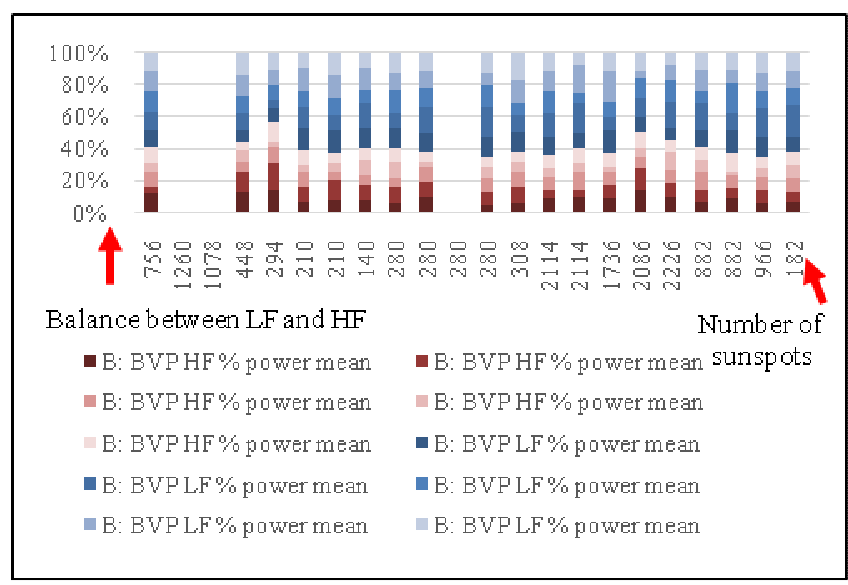

Fig.11. The dependence of the BVP HF and BVP LF signals on solar activity in emotionally labile participants. Source: [16].

At this point, we can note again that the primary aim of our effort lies in defining the impact of solar wind intensity changes on emotions, stress generation, and performance in a homogeneous sample of participants. Research of such scope has not been published thus far; one of its central benefits is the formation of a benchmark of psychophysiological values acquired from a homogeneous set of participants, the basic data range being as follows:

Age: 20 to 31

Body temperature before *)PP measurement: 35.5 to 37.5 OC

Body temperature after *)PP measurement: 35.5 to 37.0 OC Systolic blood pressure before $\left.{ }^{*}\right) \mathrm{PP}$ measurement: 100 to $180 \mathrm{mmHg}$

Diastolic blood pressure after *)PP measurement: 40 to $91 \mathrm{mmHg}$

Systolic blood pressure before *)PP measurement: 94 to $185 \mathrm{mmHg}$

Diastolic blood pressure after *)PP measurement: 48 to $99 \mathrm{mmHg}$

Number of sunspots during (Plasma waves hitting Earth) *)PP measurement: 140 to $2226 \mathrm{p} / \mathrm{cm} 3$ - number of sunspots*)PP: psychophysiological measurement with the BioGraph Infiniti device.

The output of the measurement cycles is summarized in Table 3.; while the relaxation phases Basic and Rest lasted 5 minutes, the Color and Math stages ran for two minutes only. Table 3. also shows the ranges of the measured 
psychophysiological values in the individual, consecutive phases. Importantly, special emphasis was placed on examining the LF/HF ratio; at high solar wind intensities, domination of the parasympaticus was observed. The standard deviation of this LF/HF ratio, depending on solar wind intensity changes determined by the number of sunspots, is illustrated in Fig.12. below.

The above figure indicates that, at low solar wind rates, the dispersion of the LF/HF values is more uniform, with min. 0.084 and max. 5.71. At high solar wind intensity rates (from 1,000 units), the dispersion of the $\mathrm{LF} / \mathrm{HF}$ values tends to grow and exhibits greater dynamics, namely, min. 0.056 and max. 10.94. Fig.12. simultaneously shows volatility differences in the standard deviations characteristic of the individual load phases (Basic; Color (two-minute color reading); Rest; Math (two-minute progressive subtraction of the number 7 from 1081); and Rest).

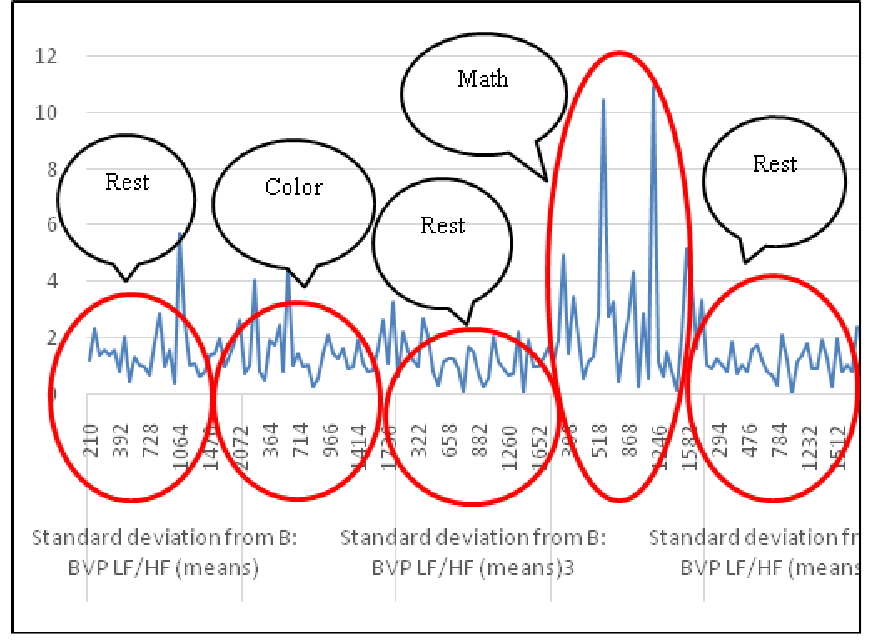

Fig.12. The BVP LF/HF standard deviation. Source: [16].

Table 3. The ranges of the measured psychophysiological values. Source: [16].

\begin{tabular}{|c|c|c|c|c|c|c|c|c|c|c|}
\hline Sequence & & asic & & Color & & Rest & & Math & & Rest \\
\hline B: BVP amplitude mean (Rel) & 0 & 30.0 & 0 & 20.6 & 0 & 22.0 & 0 & 21.9 & 0 & 21.2 \\
\hline B: BVP HR mean (beats/min) & 0 & 125.84 & 0 & 123.12 & 0 & 97.72 & 0 & 125.28 & 0 & 99.68 \\
\hline B: BVP HR std. dev. & 0 & 60.66 & 0 & 45.61 & 0 & 44.44 & 0 & 43.22 & 0 & 46.07 \\
\hline B: BVP peak freq. mean $(\mathrm{Hz})$ & 0 & 0.27 & 0 & 0.26 & 0 & 0.24 & 0 & 0.33 & 0 & 0.27 \\
\hline B: BVP IBI std. dev. (SDRR) & 0 & 366.1 & 0 & 361.47 & 0 & 366.88 & 0 & 426.15 & 0 & 363.13 \\
\hline B: BVP VLF \% power mean & 0 & 44 & 0 & 65.36 & 0 & 46.41 & 0 & 67.27 & 0 & 51.25 \\
\hline B: BVP LF \% power mean & 0 & 86.39 & 0 & 84.76 & 0 & 71.82 & 0 & 87.18 & 0 & 68.4 \\
\hline B: BVP HF \% power mean & 0 & 80.94 & 0 & 61.57 & 0 & 79.89 & 0 & 74.99 & 0 & 77.54 \\
\hline B: BVP ULF \% power mean & 0 & 16 & 0 & 31.13 & 0 & 17.7 & 0 & 32.75 & 0 & 14.98 \\
\hline B: BVP VLF total power mean & 0 & 2549.09 & 0 & 3525.78 & 0 & 12547.57 & 0 & 2874.66 & 0 & 7478.45 \\
\hline B: BVP LF Total power mean & 0 & 5142.99 & 0 & 12113.5 & 0 & 8879.18 & 0 & 5915.88 & 0 & 12536.73 \\
\hline B: BVP HF total power mean & 0 & 4464.12 & 0 & 11793.98 & 0 & 6587.36 & 0 & 7841.5 & 0 & 7094.28 \\
\hline B: BVP LF/HF (means) & 0 & 14.85 & 0 & 14.79 & 0 & 9.02 & 0 & 30.94 & 0 & 7.13 \\
\hline Total spectral power & 0 & 8629.04 & 0 & 21593.77 & 0 & 15467.89 & 0 & 13758.13 & 0 & 16747.21 \\
\hline C: EMG mean (uV) & 0 & 592.58 & 0 & 623.16 & 0 & 600.11 & 0 & 622.14 & 0 & 615.93 \\
\hline D: EMG mean (uV) & 0 & 219.63 & 0 & 325.07 & 0 & 380.12 & 0 & 613.48 & 0 & 502.14 \\
\hline E: Skin conductance mean (uS) & 0 & 17.34 & 0 & 20.58 & 0 & 18.27 & 0 & 19.19 & 0 & 17.49 \\
\hline $\mathrm{E}: \mathrm{SC}$ as $\%$ of value mean (\%) & 0 & 346.9 & 0 & 411.68 & 0 & 365.38 & 0 & 383.88 & 0 & 349.89 \\
\hline F: Temperature mean (Deg) & 0 & 36.01 & 0 & 35.98 & 0 & 36.07 & 0 & 36.04 & 0 & 36.08 \\
\hline F: Temp as $\%$ of value mean (\%) & 0 & 36.74 & 0 & 36.72 & 0 & 36.81 & 0 & 36.77 & 0 & 36.82 \\
\hline G: Resp rate mean (br/min) & 0 & 18.8 & 0 & 15.25 & 0 & 18.77 & 0 & 19.75 & 0 & 18.8 \\
\hline B\&G: (HR max-min) mean (b/min) & 0 & 51.72 & 0 & 116.92 & 0 & 84.94 & 0 & 102.94 & 0 & 92.24 \\
\hline G: Abd amplitude mean (rel) & -0.69 & 5.17 & 0 & 5.6 & 0 & 4.61 & 0 & 3.64 & 0 & 4.82 \\
\hline H: Thor amplitude mean (rel) & 0 & 4.03 & 0 & 4.43 & 0 & 3.35 & 0 & 3.03 & 0 & 2.99 \\
\hline G\&H: Abd-tho ampl diff (means) & -2.27 & 4.53 & -3.29 & 4.62 & -2.23 & 3.8 & -2.26 & 3.3 & -1.78 & 3.51 \\
\hline
\end{tabular}


Within the planned follow-up activities, we will concentrate on further examination of the investigated variables, or the HRV, EMG [32] and [33], Skins [34], Abd and Thor respiration amplitudes, which were systematically monitored in the course of the research involving a homogeneous sample of participants.

\section{APPENDIX}

The article discusses the results and evaluation of a cycle of experimental measurements performed to define the impact of solar activity upon an individual and their social interactions. The related primary research has shown that geomagnetic field changes resulting from solar eruptions affect the neurophysiological condition and status of a human being within society. The obtained knowledge and conclusions are of major importance for predictive control applications related to the management of social and economic processes (such as those within healthcare, transportation, industrial and financial markets, and supply of energy and goods).

The experiment was performed with the intention to screen out the influence of Earth's external quasistationary field.

Using a source of an offset magnetic field to simulate in laboratory conditions the effect of changes in the intensity of solar activity amplified the variation of psychophysiological parameters, depending on solar activity. The problem of the possible effect of greenhouse gases and the related impact on the ionosphere was not analyzed within the described research, mainly because, from the perspective of the time domain, ionospheric changes due to climatic variation are very slow with respect to the manifested effects of solar activity.

\section{ACKNOWLEDGMENT}

The research was funded via the National Sustainability Program, grant No. LO1401. For the actual analyses and experiments, the current infrastructure of the SIX Center was used. The authors acknowledge the help and support of the University of Defence (Brno), whose students and employees formed a homogeneous sample of participants to facilitate the actual investigation procedures.

\section{REFERENCES}

[1] Ferris, J. (2010). The brain generates an electric field that influences its own activity. Scientific American Mind, 21 (10).

[2] Unakafov, A. (2009). Analysis and modeling of the galvanic skin response spontaneous component in the context of intelligent biofeedback systems development. Measurement Science Review, 9 (2), 3641.

[3] Surkov, V., Hayakawa, M. (2014). Ultra and Extremely Low Frequency Electromagnetic Fields. Springer.
[4] International Commission on Non-Ionizing Radiation Protection (ICNIRP). (2009). Exposure to High Frequency Electromagnetic Fields, Biological Effects and Health Consequences (100 kHz - $300 \mathrm{GHz})$ : Review of the Scientific Evidence on Dosimetry, Biological Effects, Epidemiological Observations, and Health Consequences Concerning Exposure to High Frequency Electromagnetic Fields $(100 \mathrm{kHz}-300$ $\mathrm{GHz}$ ). ICNIRP, ISBN 978-3-934994-10-2.

[5] Prechter, R. (1999). The Wave Principle of Human Social Behavior and the New Science of Socionomics. New Classics Library.

[6] Miller, R.A., Miller, I. (2003). The Schumann's resonances and human psychobiology. Nexus Magazine, 10 (3).

[7] Urban, R., Holcner, V. (2010). Educating for the future challenges in military education at the beginning of the 21 st century. In Securitate şi apărare europeană în contextul crizei economico-financiare. Bucharest, Romania: Editura Universităţii Naţionale de Apărare "Carol I", ISBN 978-973-663-809-1, 128-135.

[8] Falkenstein, M., Hoormann, J., Christ, S., Hohnsbein, J. (2000). ERP components on reaction errors and their functional significance: A tutorial. Biological Psychology, 51 (2-3), 87-107.

[9] Thought Technology Ltd. Operations manual.

[10] MacLeod, C.M. (1992). The Stroop task: The "gold standard" of attentional measures. Journal of Experimental Psychology: General, 121 (1), 12-14.

[11] Hanzelka, M., Dan, J., Fiala, P., Steinbauer, M., Holcner, V. (2015). Experiments with sensing and evaluation of ionosphere changes and their impact on the human organism. In MEASUREMENT 2015: 10th International Conference on Measurement. Bratislava, Slovak Republic: Institute of Measurement Science, Slovak Academy of Sciences, 173-176.

[12] McCraty, R. (2010). Coherence: Bridging personal, social and global health. Alternative Therapies in Health and Medicine, 16 (4), 10-24.

[13] NASA. (2015). The solar wind. http://solarscience. msfc.nasa.gov/SolarWind.shtml

[14] Krampen, G. (2006). Änderungssensitive Symptomliste zu Entspannungserleben, Wohlbefinden, Beschwerden und Problembelastungen (ASS-SYM). Göttingen: Hogrefe.

[15] Dan, J. (2009). Lüscher - Color - Diagnostik. Tschechische Version. In Lüscher - Color Diagnostik. Brunnen/Schweiz, 1.- 4.10.2009. 2009.

[16] Štastný, P. (2015). Solar Activity : Analytical Software in xls. Brno.

[17] Berens, L., Nardi, D. (1999). The Sixteen Personality Types: Descriptions for Self-Discovery. Radiance House.

[18] Dan, J., Edelmann, A. (2013). Max Lüschers Color Test. 
[19] Woodworth, R.S., Schlosberg, H. (1959). Experimentálna psychológia (Experimental Psychology). Bratislava, Slovak Republic: VEDA, the Publishing House of the Slovak Academy of Sciences, 149-176. (in Slovak)

[20] McCraty, R., Atkinson, M., Tiller, W.A., Rein, G., Watkins, A.D. (1995). The effects of emotions on short-term power spectrum analysis of heart rate variability. American Journal of Cardiology, 76 (14), 1089-1093.

[21] Umetani, K., Singer, D.H., McCraty, R., Atkinson, M. (1998). Twenty-four hour time domain heart rate variability and heart rate: Relations to age and gender over nine decades. Journal of the American College of Cardiology, 31 (3), 593-601.

[22] Levy, B.R., Slade, M.D., Kunkel, S.R., Kasl, S.V. (2002). Longevity increased by positive selfperceptions of aging. Journal of Personality and Social Psychology, 83 (2), 261-270.

[23] Lindmark, S., Lonn, L., Wiklund, U., Tufvesson, M., Olsson, T., Eriksson, J.W. (2005). Dysregulation of the autonomic nervous system can be a link between visceral adiposity and insulin resistance. Obesity Research, 13 (4), 717-728.

[24] Saul, J.P., Arai, Y., Berger, R.D., Lilly, L.S., Colucci, W.S., Cohen, R.J. (1988). Assessment of autonomic regulation in chronic congestive heart failure by heart rate spectral analysis. American Journal of Cardiology, 61 (15), 1292-1299.

[25] Beauchaine, T. (2001). Vagal tone, development, and Gray's motivational theory: Toward an integrated model of autonomic nervous system functioning in psychopathology. Development and Psychopathology, 13 (2), 183-214.

[26] Thayer, J.F., Hansen, A.L., Saus-Rose, E., Johnsen, B.H. (2009). Heart rate variability, prefrontal neural function, and cognitive performance: The neurovisceral integration perspective on selfregulation, adaptation, and health. Annals of Behavioral Medicine, 37 (2), 141-153.
[27] McCrea, I.W., Jones, T.B., Schlegel, K., Tuomo, N. (1991). COSCAT, a new auroral radar facility on 930 $\mathrm{MHz}$ - system description and first results. Annales Geophysicae, 9, 461-469.

[28] Lehrer, P., Vaschillo, E., Lu, S.E., Eckberg, D. Vaschillo, B., Scardella, A., Habib, R. (2006). Heart rate variability biofeedback: Effects of age on heart rate variability, baroreflex gain, and asthma. Chest, 129 (2), 278-284.

[29] Foreman, R. (1997). Organisation of visceral input. In Anesthesia: Biologic Foundations. Lippincott-Raven Publishers, 663-683.

[30] Siegel, G., Ebeling, B.J., Hofer, H.W., Nolte, J., Roedel, H., Klubendorf, D. (1984). Vascular smooth muscle rhythmicity. In Mechanisms of Blood Pressure Waves. Japan Scientific Societies Press; Springer, 319338.

[31] Baselli, G., Cerutti, S., Badilini, F., Biancardi, L., Porta, A., Pagani, M., Lombardi, F., Rimoldi, O., Furlan, R., Malliani, A. (1994). Model for the assessment of heart period variability interactions of respiration influences. Medical and Biological Engineering and Computing, 32 (2), 143-152.

[32] Phinyomark, A., Limsakul, C., Phukpattaranont, P. (2011). Application of wavelet analysis in EMG feature extraction for pattern classification. Measurement Science Review, 11 (2), 45-52.

[33] Mishra, P., Singla, S.K. (2013). Artifact removal from biosignal using fixed point ICA algorithm for preprocessing in biometric recognition. Measurement Science Review, 13 (1), 7-11.

[34] Unakafov, A. (2009). Analysis and modeling of the galvanic skins response spontaneous component in the context of intelligent biofeedback systems development. Measurement Science Review, 9 (2), 3641.
Received July 3, 2016. Accepted January 24, 2017. 